\title{
A Stress-Dependent Hysteresis Model for PZT-Based Transducers
}

\author{
Brian L. Ball ${ }^{1}$ and Ralph C. Smith ${ }^{2}$ \\ Center for Research in Scientific Computation \\ Department of Mathematics \\ North Carolina State University \\ Raleigh, NC 27695
}

\begin{abstract}
A ferroelastic switching model for single crystal piezoceramic compounds is developed. The model is based on a phenomenological Landau-Devonshire type thermodynamic theory for the materials. The model incorporates externally applied electric fields and compressive stress inputs to the crystals and models the $90^{\circ}$ and $180^{\circ}$ ferroelastic and ferroelectric switching induced by the inputs. Properties of the ferroelastic model are qualitatively similar to experimental PLZT data.
\end{abstract}

Keywords: Hysteresis, ferroelastic switching, ferroelectric switching, Landau free-energy

\section{Introduction}

Lead zirconate titanate (PZT)-based transducers have a variety of high-performance applications ranging from high-speed switching to nanoscale positioning. Stress-biased actuators such as THin layer UNimorph ferroelectric Driver and sEnoR (THUNDER) and Reduced And INternally Biased Oxide Wafer (Rainbow) exhibit enhanced displacements due to a variety of mechanisms including stress-induced domain alignment $[7,4,2]$. At high stresses, ferroelastic switching must be incorporated into models to maintain the accuracy needed for high-performance applications. Inclusion of ferroelastic switching is also necessary to model the stress-dependent electromechanical behavior measured in THUNDER transducers [7].

This paper develops a ferroelastic switching model for single crystal ferroceramic compounds that is based on a phenomenological Landau-Devonshire type thermodynamic theory of $\mathrm{PbTiO}_{3}$ developed in [1]. The model incorporates externally applied electric field and compressive stress inputs to the materials and models the $90^{\circ}$ and $180^{\circ}$ ferroelastic and ferroelectric switching induced by the inputs. Properties of the ferroelastic model are investigated and shown to be qualitatively analogous to experimental PLZT data reported in [3]. Work to directly compare the model to experimental data is ongoing.

\section{Ferroelectric and Ferroelastic Switching Mechanisms}

In this section, we review the ferroelectric and ferroelastic switching mechanisms in ferroelectric compounds which we illustrate in the context of $\mathrm{PbTiO}_{3}$. As outlined in [3], lead titanate $\left(\mathrm{PbTiO}_{3}\right)$ has an $\mathrm{ABO}_{3}$ perovskite oxide structure. For temperatures above the Curie temperature $T_{c}$, the structure of $\mathrm{PbTiO}_{3}$ is cubic whereas for $T<T_{c}$, the structure of lead titanate is either tetragonal, orthorhombic or rhombohedral. To simplify our model, we consider the two dimensional case where the polarization has values only in the 1 and 3 directions, denoted by $P_{1}$ and $P_{3}$, respectively. Therefore, we shall limit our discussion to the paraelectric cubic structure and the ferroelectric tetragonal structure for $\mathrm{PbTiO}_{3}$ which are illustrated in Figure 1.

\footnotetext{
${ }^{1}$ Email: blball@unity.ncsu.edu, Telephone: (919) 332-7957

${ }^{2}$ Email: rsmith@eos.ncsu.edu, Telephone: (919) 515-7552
} 


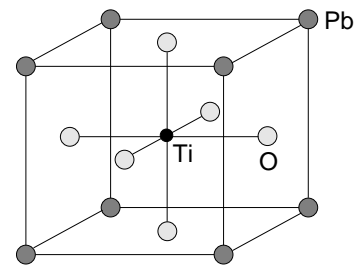

(a)

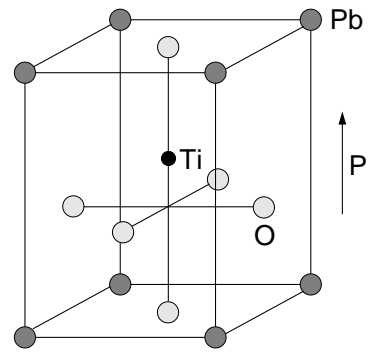

(b)

Figure 1. (a) High temperature paraelectric cubic form of lead titanate, and (b) low temperature ferroelectric tetragonal form of lead titanate.

Ferroelectric switching is induced by the application of an electric field that is larger in magnitude than the coercive field $E_{c}$. This causes the central $\mathrm{Ti}^{+4}$ ion to relocate to a new equilibrium position, resulting in a $180^{\circ}$ change in polarization that is parallel to the applied field as depicted in Figure 2(a). Ferroelastic switching is caused by the application of a stress that is larger in magnitude than the coercive stress $\sigma_{c}$. The resulting equilibrium position of the $\mathrm{Ti}^{+4}$ ion results in a $90^{\circ}$ change in polarization that is perpendicular to the applied stress as illustrated in Figure 2(b). The ferroelectric and ferroelastic switching mechanisms cause a hysteretic relationship between input fields $E$ and $\sigma$ and output polarization $P$ and strains $\varepsilon$. Details regarding a typical ferroelectric and ferroelastic response of soft piezoelectric ceramics can be found in [3].

Figures 3(a) and 3(b) illustrate the relationship between an externally applied field and the polarization and strains induced in a soft PZT. At point A, the electric field is sufficiently strong so that all the dipoles form one domain that is aligned in the direction of the applied field. As the field is decreased in magnitude it approaches the coercive field. In the region around point B, $180^{\circ}$ switching occurs. This often includes $90^{\circ}$ switching as indicated by the presence of a negative strain at point B as depicted in Figure 3(b). At point C, all the domains have switched and the dipoles form one domain that is aligned in the direction of the electric field. At point $\mathrm{C}$, the polarization is opposite to that at point A whereas the strains have the same value. As the field is again reduced in magnitude, $90^{\circ}$ switching occurs at point $\mathrm{D}$ and rapidly continues back to point $\mathrm{A}$ where the full $180^{\circ}$ switch has occurred and the dipoles are again aligned with the applied electric field.

Figures 3(c) and 3(d) illustrate the relationship between an externally applied stress and the polarization and strains induced in the soft PLZT. At point A, the dipoles are aligned in the positive 3 direction and the material acts as one domain. As the compressive stress is increased in magnitude, it approaches the coercive stress. In the region around point $\mathrm{B}, 90^{\circ}$ switching occurs and the dipoles begin to align perpendicular to the direction of the applied stress. This is indicated by the presence of a negative strain at point B in Figure 3(d). As the stress is reduced in magnitude, the material stays poled perpendicular to the applied stress.

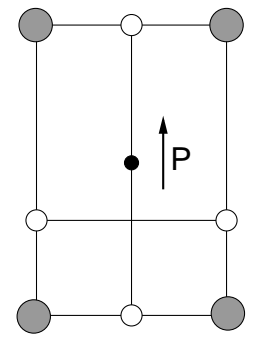

(a)

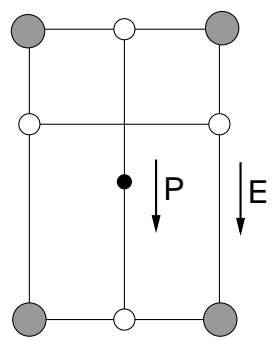

)

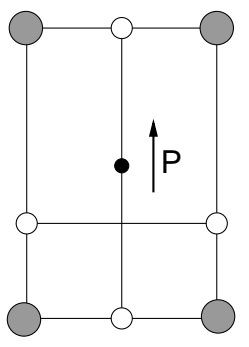

(b)

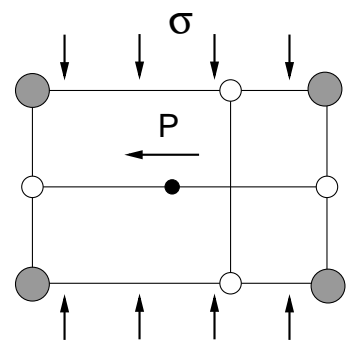

Figure 2. (a) $180^{\circ}$ switch induced by an applied electric field, and (b) $90^{\circ}$ switch induced by an applied stress. 


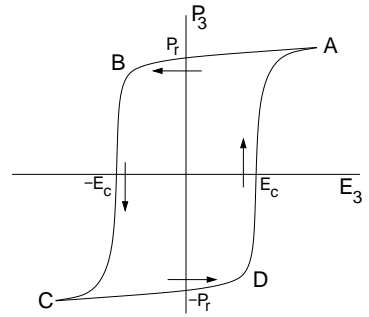

(a)

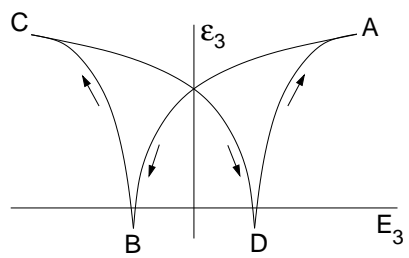

(b)

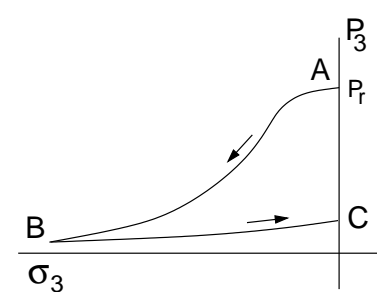

(c)

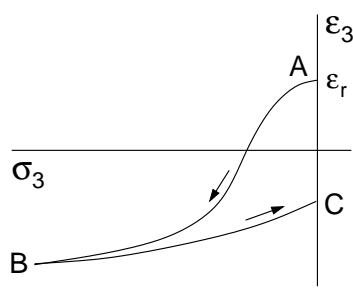

(d)

Figure 3. (a) Hysteretic field-polarization relation for bulk PLZT, (b) field-strain behavior of PLZT, (c) stress-polarization relation for PLZT, and (d) stress-strain behavior of PLZT.

\section{Ferroelectric Switching Model}

We summarize here a ferroelectric switching model which characterizes the hysteretic field-polarization and field-strain relations depicted in Figures 3(a) and 3(b). As detailed in [8], macroscopic models for the hysteretic electric field-polarization relation in piezoceramics are typically based on phenomenological principles, thermodynamic tenets, or energy formulations employed in concert with homogenization techniques. Preisach theory has the advantage of providing a mathematical model quantifying hysteresis when the underlying physics is poorly understood. However, the resulting models tend to have a large number of nonphysical parameters which is undesirable for parameter identification. Also, the original Preisach theory does not accommodate reversible effects or variable operating temperatures, and the inclusion of such effects significantly diminishes the efficiency of extended models.

The macroscopic ferroelectric polarization model summarized here was developed in [8] through a combination of free energy principles at the lattice level and stochastic homogenization techniques to incorporate material nonhomogeneaties and variable effective fields. As detailed in [8], the resulting macroscopic model guarantees closure of biased minor loops in quasistatic operating regimes and incorporates reversible effects. The model is sufficiently low-order to facilitate real-time control implementation as illustrated for an analogous magnetic model in [6]. Boltzmann principles are used to construct the Helmholtz energy through an internal energy balance of positive and negative dipole configurations and entropy effects. A Gibbs relation $G$ quantifying the change in the energy landscape due to an applied field is provided by the inclusion of the electrostatic work term and in the limit of low thermal activation, the necessary condition $\frac{\partial G}{\partial P}=0$ gives the local polarization.

\subsection{Local Average Polarization}

For fixed temperatures, we employ the theory of [8] in which it is illustrated that a reasonable expression for the Helmholtz energy is the piecewise quadradic relation

$$
\psi(P)= \begin{cases}\frac{1}{2} \eta\left(P+P_{R}\right)^{2} & , \quad P \leq-P_{I} \\ \frac{1}{2} \eta\left(P-P_{R}\right)^{2} & , \quad P \geq P_{I} \\ \frac{1}{2} \eta\left(P_{I}-P_{R}\right)\left(\frac{P^{2}}{P_{I}}-P_{R}\right) & , \quad|P|<P_{I}\end{cases}
$$

where $P_{I}$ and $P_{R}$ denote the positive inflection point and polarization at which the minimum occurs. The resulting Gibbs free energy is derived by combining the potential energy of a dipole in the field with the Helmholtz free energy throughout the lattice to yield

$$
G=\psi-E P .
$$

It is illustrated in [8] that under the assumption of low thermal activation, the local average polarization $\bar{P}$, at fixed temperature, can be derived from the necessary condition that $\frac{\partial G}{\partial P}=0$. For computational efficiency, $\bar{P}$ is formulated as

$$
\bar{P}=\frac{E}{\eta}+P_{R} \Delta
$$


where $\Delta=1$ if evaluating on the upper branch of the hysteresis kernel and $\Delta=-1$ if evaluating on the lower branch. Details regarding numerical algorithms for constructing $\Delta$ as well as the development of a relation for $\bar{P}$ which incorporates frequency and thermal effects are provided in [8].

\subsection{Macroscopic Polarization Model}

Nonuniformities in the lattice structure of PZT produce a distribution of Helmholtz and Gibbs energy profiles which can be manifested as variations in the local coercive field and local remanent polarization and can produce differing saturation behavior after dipole switching. Other variations can be produced by stress nonhomogeneities, nonuniform lattice orientations across grain boundaries, and crystalline anisotropies.

To incorporate lattice variability, we consider the coercive field $E_{c}$ to be a manifestation of an underlying distribution $\nu_{1}\left(E_{c}\right)$ rather than fixed values which is typically assumed for single crystals having a uniform lattice structure. To create a macroscopic model for the polarization, we also consider the variation of effective fields in the material. As detailed in [8], an applied field in a ferroelectric material is augmented by fields generated by neighboring dipoles which produce nonhomogeneous effective fields in the material. This, along with various other processes, produces variations in the applied field that can significantly alter the resulting polarization. To incorporate these variations, we consider the effective field to be distributed about the applied field by a density $\nu_{2}\left(E_{e}\right)$.

The introduction of variations in the effective field produces domain switching in advance of the remanence point in accordance with observations from experimental data. The complete macroscopic polarization model, as derived in [8], for nonhomogeneous, polycrystalline materials with variable effective fields is given by

$$
[P(E)]=\int_{0}^{\infty} \int_{-\infty}^{\infty}\left[\bar{P}\left(E+\varepsilon ; E_{c}\right)\right] \nu_{1}\left(E_{c}\right) \nu_{2}\left(E_{e}\right) d E_{e} d E_{c}
$$

where $\bar{P}$ is defined by (3). Note that (4) does not incorporate ferroelastic coupling so this formulation of the polarization model should be restricted to low stress regimes. As shown in $[4,8]$, the ferroelectric model accurately and efficiently models the field-polarization behavior depicted in Figure 3(a). However, the model does not describe the stress dependent processes including those illustrated in Figures 3(c) and 3(d).

\section{Ferroelastic Switching Model}

Here we introduce a ferroelastic switching model which quantifies the hysteretic stress-polarization and stressstrain relationships depicted in Figures 3(c) and 3(d) and also characterizes the hysteretic field-polarization and field-strain relationships depicted in Figures 3(a) and 3(b). The ferroelastic model is essentially a extension of the ferroelectric model described in the previous section and is based on a phenomenological Landau-Devonshire energy relation as employed in [1].

To model the internal free-energy of a lead titanate crystal, we use a 2D Landau-Devonshire free-energy for materials exhibiting second-order phase transitions. We consider electric field and stress inputs $(\vec{E}, \vec{\sigma})$ and polarization and strain outputs $(\vec{P}, \vec{\varepsilon})$ to have components in the 1 and 3 directions. The Landau-Devonshire free-energy density is taken to be

$$
\psi_{P}(\vec{P})=\alpha_{1} P_{1}^{2}+\alpha_{3} P_{3}^{2}+\alpha_{11} P_{1}^{4}+\alpha_{33} P_{3}^{4}+\alpha_{31} P_{3}^{2} P_{1}^{2}
$$

and is a function of the two allowed polarization values $P_{1}$ and $P_{3}$. The coefficients are chosen so that $\alpha_{i j}>0$ and $\alpha_{i}<0$ below the Curie temperature and can be related to physical properties of the $\mathrm{PbTiO}_{3}$ crystal such as the remanence polarization $P_{R}$ and coercive field $E_{c}$ by equations (17) and (18). The electromechanical coupling energy density is given by

$$
\psi_{e s}(\vec{P}, \vec{\varepsilon})=-a_{1} \varepsilon_{1} P_{1}-a_{3} \varepsilon_{3} P_{3}-a_{13} \varepsilon_{1} P_{3}-a_{31} \varepsilon_{3} P_{1}-q_{1} \varepsilon_{1} P_{1}^{2}-q_{3} \varepsilon_{3} P_{3}^{2}-q_{13} \varepsilon_{1} P_{3}^{2}-q_{31} \varepsilon_{3} P_{1}^{2}
$$

where $a_{i j}$ are the piezoelectric coupling coefficients and $q_{i j}$ are the electrostrictive coupling coefficients. Ignoring shear effects, the elastic free-energy density is

$$
\psi_{e l}(\vec{\varepsilon})=\frac{1}{2} Y_{1} \varepsilon_{1}^{2}+\frac{1}{2} Y_{3} \varepsilon_{3}^{2}
$$




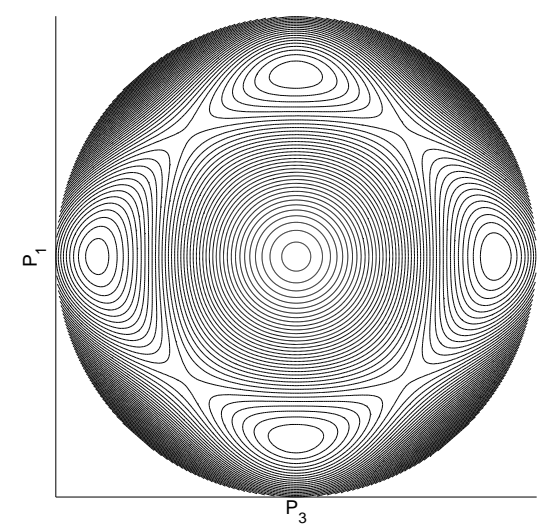

Figure 4. Gibbs free energy landscape with four local minimum for the 4 allowed polarizations: $\pm P_{1}, \pm P_{3}$.

where $Y_{i}$ are the elastic coefficients. The total free-energy density is then given by

$$
\psi(\vec{P}, \vec{\varepsilon})=\psi_{P}(\vec{P})+\psi_{e s}(\vec{P}, \vec{\varepsilon})+\psi_{e l}(\vec{\varepsilon}) .
$$

The work due to an externally applied electric field and an applied stress is incorporated by employing a Gibbs energy of the form

$$
G(\vec{E}, \vec{P}, \vec{\sigma}, \vec{\varepsilon})=\psi(\vec{P}, \vec{\varepsilon})-\vec{E} \cdot \vec{P}-\vec{\sigma} \cdot \vec{\varepsilon} .
$$

The necessary conditions for minimizing the Gibbs energy are $\frac{\partial G}{\partial \varepsilon_{1}}=0, \frac{\partial G}{\partial \varepsilon_{3}}=0, \frac{\partial G}{\partial P_{1}}=0$ and $\frac{\partial G}{\partial P_{3}}=0$. These conditions specify how the internal polarization and strains change to minimize the internal energy when an external force is applied and thermal activation is negligable. The condition $\frac{\partial G}{\partial \varepsilon_{1}}=0$ implies that

$$
\varepsilon_{1}=Y_{1}^{-1}\left(\sigma_{1}+a_{1} P_{1}+a_{13} P_{3}+q_{1} P_{1}^{2}+q_{13} P_{3}^{2}\right)
$$

and $\frac{\partial G}{\partial \varepsilon_{3}}=0$ implies that

$$
\varepsilon_{3}=Y_{3}^{-1}\left(\sigma_{3}+a_{3} P_{3}+a_{31} P_{1}+q_{3} P_{3}^{2}+q_{31} P_{1}^{2}\right) .
$$

These relations allow us to determine explicit expressions for the strains. In a manner analogous to that employed in [5] for a stress-free state, we substitute equations (10) and (11) into the Gibbs energy (9) to directly couple the stress and the polarization. This yields

$$
G(\vec{E}, \vec{P}, \vec{\sigma})=\widehat{\psi}(\vec{P}, \vec{\sigma})-\vec{E} \cdot \vec{P}
$$

where

$$
\begin{aligned}
\widehat{\psi}(\vec{P}, \vec{\sigma})= & \gamma_{1} P_{1}^{4}+\gamma_{2} P_{3}^{4}+\gamma_{3} P_{3}^{2} P_{1}^{2}+\gamma_{4} P_{1}^{3}+\gamma_{5} P_{3}^{3}+\gamma_{6} P_{1}^{2} P_{3}+\gamma_{7} P_{1} P_{3}^{2} \\
& +\gamma_{8} P_{1}^{2}+\gamma_{9} P_{3}^{2}+\gamma_{10} P_{3} P_{1}+\gamma_{11} P_{1}+\gamma_{12} P_{3}+\gamma_{13} .
\end{aligned}
$$

The Gibbs energy at zero stress and zero electric field is plotted in Figure 4. There are four local minimum each corresponding to the four allowed polarization states $\pm P_{1}$ and $\pm P_{3}$. Given an input pair $(\vec{E}, \vec{\sigma})$, the polarization is then specified by the minimum

$$
\min _{P_{1}, P_{3}} G(\vec{E}, \vec{P}, \vec{\sigma})=\min _{P_{1}, P_{3}} \widehat{\psi}(\vec{P}, \vec{\sigma})-\vec{E} \cdot \vec{P} .
$$

The ferroelastic model thus quantifies the $\left(P_{1}, P_{3}\right)$ pair that minimizes the total energy of the system and the corresponding strain pair $\left(\varepsilon_{1}, \varepsilon_{3}\right)$ are computed by equations (10) and (11).

As mentioned previously, several of the coefficients in the ferroelastic model are related to physical material properties, thus allowing a means of determining their values. The remenant polarization $P_{R}$ and coercive field $E_{c}$ are computed by considering a stress free Gibbs energy

$$
G(\vec{E}, \vec{P})=\psi(\vec{P})-\vec{E} \cdot \vec{P}
$$


Furthermore, the conditions $P_{1}=0$ and $\frac{\partial G}{\partial P_{3}}=0$ imply that

$$
4 \alpha_{33} P_{3}^{3}+2 \alpha_{3} P_{3}-E_{3}=0 .
$$

As outlined in [9], equation (16) has three real roots, of which at least two are equal if the discriminant is 0. The coercive field value for which this property is satisfied is

$$
E_{c}=\sqrt{\frac{-4^{3} \alpha_{3}^{3}}{6^{3} \alpha_{33}}} .
$$

When $E_{3}=0$, the remenant polarization determined from solving (16) is given by

$$
P_{R}=\sqrt{\frac{-\alpha_{3}}{2 \alpha_{33}}} .
$$

Equations (17) and (18) provide a system of two equations and two unknowns sufficient to solve for $\alpha_{3}$ and $\alpha_{33}$ if $E_{c}$ and $P_{R}$ are known. A similar processes may be applied to find $\alpha_{1}$ and $\alpha_{11}$ if the energy landscape is known to be nonsymmetric.

\section{Model Results}

To numerically illustrate the properties of the ferroelastic model, an applied electric field $E_{3}$ and an applied compressive stress $\sigma_{3}$ are applied in the 3 -direction. The resulting polarization $P_{3}$ and the strain $\varepsilon_{3}$ are computed and plotted in Figures 5-7. Figure 5 illustrates the $E_{3}-P_{3}$ and $E_{3}-\varepsilon_{3}$ behavior with no compressive stress. The $E_{3}-P_{3}$ and $E_{3}-\varepsilon_{3}$ behavior with a constant applied stress of $-20 \mathrm{MPa}$ is depicted in Figure 6 . Figure 7 contains a plot of $P_{3}$ and $\varepsilon_{3}$ verses an applied compressive stress at zero applied electric field. All simulations were performed utilizing an unconstrained nonlinear optimization code with an analytic gradient and Hessian to decrease computational effort.

The model yields relatively similar results when there is no applied external stress. However, as shown in [8], the ferroelectric model has a piecewise linear hysteron where as the $E_{3}-P_{3}$ hysteron derived from the ferroelastic model is not linear, as shown in Figure 5(a). Figure 6(a) illustrates how at higher constant compressive stresses, the polarization remains in the $90^{\circ}$ well longer than at lower compressive stresses during the $180^{\circ}$ switch. This is also verified by the negative strain exhibited in Figure 6(b).

Figure 7 (a) illustrates how $90^{\circ}$ switching occurs as the compressive stress increases beyond the coercive stress. As the crystal is loaded in the 3-direction, dipoles initially oriented in the 3-direction switch to align perpendicular to the applied stress and remain in the 1-direction during unloading. This is also verified by

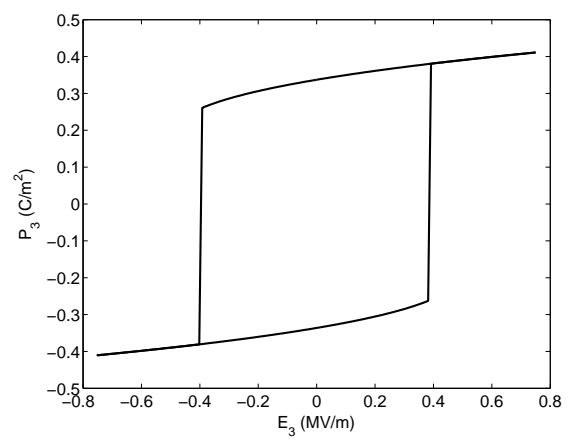

(a)

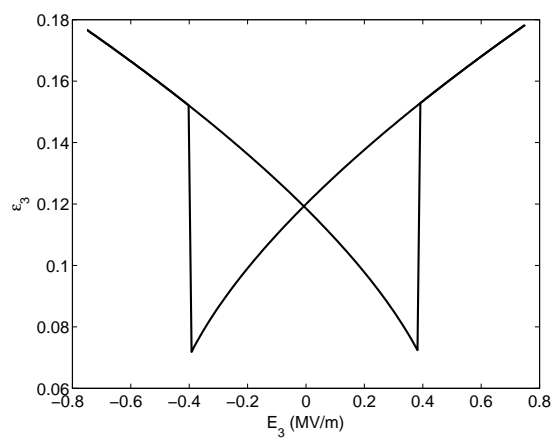

(b)

Figure 5. Ferroelectric behavior with no prestress. (a) $E_{3}-P_{3}$ relation, and (b) $E_{3}-\varepsilon_{3}$ relation. 


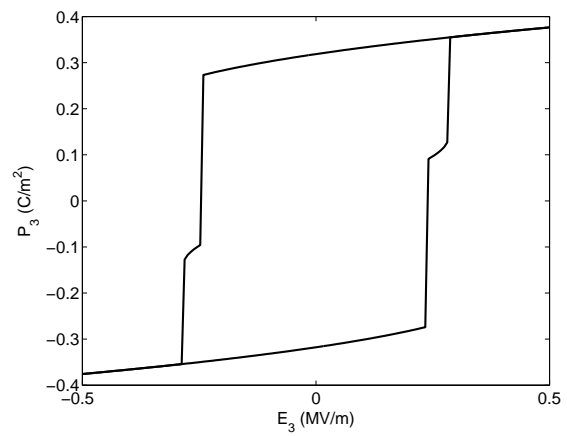

(a)

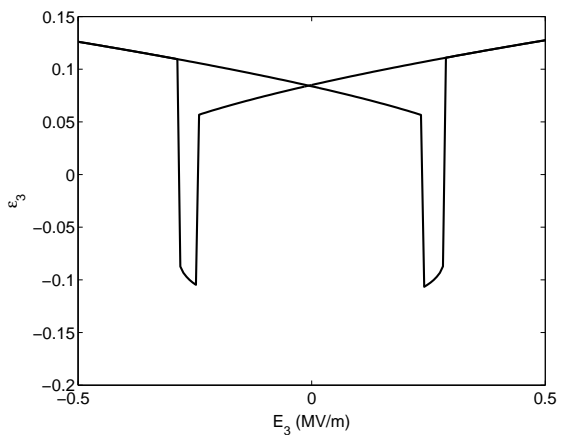

(b)

Figure 6. Ferroelectric behavior with a prestress of $-20 \mathrm{MPa}$. (a) $E_{3}-P_{3}$ relation, and (b) $E_{3}-\varepsilon_{3}$ relation.

Figure 7(b) where the crystal has a negative strain after loading. All plots in Figures 5-7 are indicative of single crystal behavior and are similar to the experimental results for PLZT at varying applied stresses that are presented in [3]. Work to identify model parameters that match the experimental data is ongoing.

\section{Concluding Remarks}

The ferroelastic model presented in this paper characterizes the single crystal $\mathrm{PbTiO}_{3}$ response to an applied electric field and an applied compressive stress. The ferroelastic model is derived using a 2D Landau-Devonshire free energy for the Helmholtz free energy. Minimization of the Gibbs energy is then employed to model the $90^{\circ}$ and $180^{\circ}$ domain switching that occurs in soft PZT ceramics due to electric and stress inputs. The model is an extension of the ferroelectric model presented in [8] and is based on the phenomenological thermodynamic theory developed in [1] and [5]. Work to describe the bulk behavior of PZT and compare to experimental data is ongoing.

\section{Acknowledgements}

This research was supported by the Air Force Office of Scientific Research under the grant AFOSR-F49620-01$1-0107$.

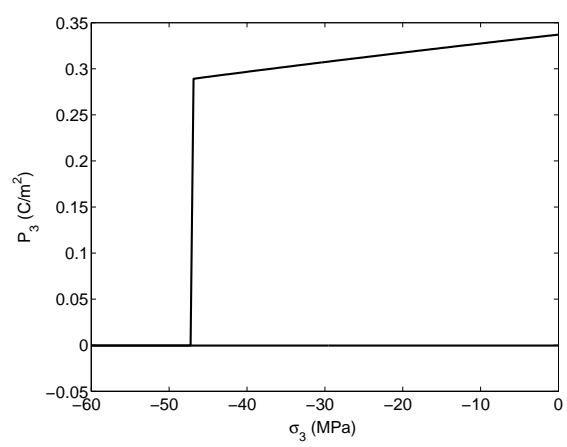

(a)

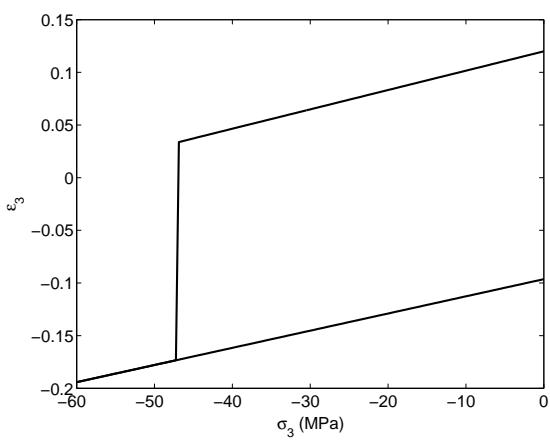

(b)

Figure 7. Ferroelastic switching at $0 \mathrm{MV} / \mathrm{m}$. (a) $\sigma_{3}-P_{3}$ behavior, and (b) $\sigma_{3}-\varepsilon_{3}$ behavior. 


\section{References}

[1] M.J. Haun, E. Furman, S.J. Jang, H.A. McKinstry and L.E. Cross, "Thermodynamic theory of $\mathrm{PbTiO}_{3}$," Journal of Applied Physics, 62(8), pp. 3331-3338, 1987.

[2] G. Li, E. Furman and G.H. Haertling, "Stress-enhanced displacements in PLZT rainbow actuators," Journal of the American Ceramic Society, 80, pp. 1382-1388, 1997.

[3] C.S. Lynch, "The effect of uniaxial stress on the electro-mechanical response of 8/65/35 PLZT," Acta Materialia, 44(10), pp. 4137-4148, 1996.

[4] K. Mossi, Z. Ounaies, R.C. Smith and B.L. Ball, "Prestressed curved actuators: characterization and modeling of their piezoelectric behavior," Proceedings of the SPIE, Vol. 5053, pp. 423-435, Smart Structures and Materials, San Diego, CA, 2003.

[5] Shinji Nambu and Djuniadi A. Sagala, "Domain formation and elastic long-range interaction in ferroelectric perovskites," Physical Review B, 50(9), pp. 5838-5847, 1994.

[6] J. Nealis and R.C. Smith, "Robust control of magnetostrictive actuator," Proceedings of the SPIE, Vol. 5049, pp. 221-232, Smart Structures and Materials, San Diego, CA, 2003.

[7] Z. Ounaies, K. Mossi, R.C. Smith and J. Bernd, "Low-field and high-field characterization of THUNDER actuators," Proceedings of the SPIE, Vol. 4333, pp. 399-407, Smart Structures and Materials, San Diego, CA, 2001.

[8] R.C. Smith, S. Seelecke, Z. Ounaies and J. Smith, "A free energy model for hysteresis in ferroelectric materials," Journal of Intelligent Material Systems and Structures, 14(11), pp. 719-739, 2003.

[9] D. Zwillinger, Editor-in-Chief, CRC Standard Mathematical Tables and Formulae, 30th Edition, CRC Press, Boca Raton, 1996. 